\title{
Prospective analysis of adrenal function in patients with acute exacerbations of COPD: the Reduction in the Use of Corticosteroids in Exacerbated COPD (REDUCE) trial
}

\author{
Philipp Schuetz, Jörg D Leuppi' ${ }^{1}$, Roland Bingisser², Michael Bodmer'2, Matthias Briel ${ }^{3,14}$ \\ Tilman Drescher ${ }^{4}$, Ursula Duerring ${ }^{4}$, Christoph Henzen ${ }^{5}$, Yolanda Leibbrandtt ${ }^{6}$, Sabrina Maier ${ }^{1}$, \\ David Miedinger ${ }^{1}$, Beat Mueller, Andreas Scherr ${ }^{7,8}$, Christian Schindler ${ }^{9}$, Rolf Stoeckli ${ }^{4}$, \\ Sebastien Viatte ${ }^{6,10}$, Christophe von Garnier ${ }^{11}$, Michael Tamm ${ }^{8}$ and Jonas Rutishauser ${ }^{12,13}$ \\ Department of Endocrinology, Diabetology, and Metabolism, Medical University Clinic, Kantonsspital, Tellstrasse \\ H7, CH-5001 Aarau, Switzerland, 'Medical University Clinic, Kantonsspital Baselland, CH-4410 Liestal, Switzerland, \\ ${ }^{2}$ Interdisciplinary Emergency Department, ${ }^{3}$ Basel Institute for Clinical Epidemiology and Biostatistics and ${ }^{4}$ Clinic of \\ Endocrinology, Diabetology, and Metabolism, University Hospital, CH-4031 Basel, Switzerland, ${ }^{5}$ Department of \\ Internal Medicine, Kantonsspital, CH-6000 Luzern 16, Switzerland, ${ }^{6}$ Department of Medicine, Clinic of Internal \\ Medicine, Hospital Center, CH-2501 Biel-Bienne, Switzerland, ${ }^{7} \mathrm{Clinic}$ of Internal Medicine, University Hospital, \\ CH-4013 Basel, Switzerland, ${ }^{8}$ Clinic of Pneumology, University Hospital, Petersgraben 4, CH-4031 Basel, Switzerland, \\ ${ }^{9}$ Swiss Tropical and Public Health Institute, University of Basel, Socinstrasse 57, CH-4051 Basel, Switzerland, \\ ${ }^{10}$ Arthritis Research UK Epidemiology Unit, Manchester Academic Health Science Center, The University of \\ Manchester, Manchester, UK, ${ }^{11}$ Respiratory Medicine Clinic, University Hospital Bern, Inselspital, CH-3010 Bern, \\ Switzerland, ${ }^{12}$ Medical University Clinic, Kantonsspital Baselland, CH-4101 Bruderholz, Switzerland, ${ }^{13}$ Biozentrum, \\ Klingelbergstrasse 50/70, University of Basel, CH-4056 Basel, Switzerland and ${ }^{14}$ Department of Clinical \\ Epidemiology and Biostatistics, McMaster University, Hamilton, Ontario, Canada
}

Correspondence should be addressed to J Rutishauser Email j.rutishauser@unibas.ch

\begin{abstract}
Objective: To analyze prospectively the hypothalamic-pituitary-adrenal (HPA) axis and clinical outcome in patients treated with prednisone for exacerbated chronic obstructive pulmonary disease (COPD).

Design: Prospective observational study.

Subjects and methods: Patients presenting to the emergency department were randomized to receive $40 \mathrm{mg}$ prednisone daily for 5 or 14 days in a placebo-controlled manner. The HPA axis was longitudinally assessed with the $1 \mu \mathrm{g}$ corticotropin test and a clinical hypocortisolism score at baseline, on day 6 before blinded treatment, at hospital discharge, and for up to 180 days of follow-up. Prednisone was stopped abruptly, irrespective of the test results. Patients discharged with pathological test results received instructions about emergency hydrocortisone treatment.

Results: A total of 311 patients were included in the analysis. Mean basal and stimulated serum total cortisol levels were highest on admission ( $496 \pm 398$ and $816 \pm 413 \mathrm{nmol} / \mathrm{l}$ respectively) and lowest on day 6 ( $235 \pm 174$ and $453 \pm 178 \mathrm{nmol} / \mathrm{l}$ respectively). Pathological stimulation tests were found in $63,38,9,3$, and $2 \%$ of patients on day 6 , at discharge, and on days 30, 90, and 180 respectively, without significant difference between treatment groups. Clinical indicators of hypocortisolism did not correlate with stimulation test results, but cortisol levels were inversely associated with re-exacerbation risk. There
\end{abstract} were no hospitalizations or deaths as a result of adrenal crisis.

Conclusion: Dynamic changes in the HPA axis occur during and after the treatment of acute exacerbations of COPD. In hypocortisolemic patients who were provided with instructions about stress prophylaxis, the abrupt termination of prednisone appeared safe. 


\section{Introduction}

The use of systemic glucocorticoids is a cornerstone in the treatment of acute exacerbations of chronic obstructive pulmonary disease (COPD) (1). Many COPD patients experience repeated exacerbations and are therefore burdened with the acute and cumulative adverse effects of this therapy. Thus, exposure to glucocorticoids should be minimized. Recently, the Reduction in the Use of Corticosteroids in Exacerbated COPD (REDUCE) randomized controlled trial demonstrated the noninferiority of a 5 -day as compared to a 14-day treatment course of $40 \mathrm{mg}$ oral prednisone daily with respect to re-exacerbation during 6 months of follow-up (2).

Treatment with therapeutic doses of glucocorticoids suppresses the hypothalamic-pituitary-adrenal (HPA) axis, which results in impaired adrenal reserve and hypocortisolism after the exogenous steroid supply has been stopped (3). Whether duration of glucocorticoid therapy and/or cumulative dose predict adrenal insufficiency $(4)$ or not $(5,6)$ is controversial. Stimulation tests are usually needed to assess the HPA axis because basal cortisol levels may be within the normal range, particularly in secondary adrenal insufficiency (3), and they do not reliably reflect adrenal reserve (5). The insulin tolerance test is the generally agreed upon reference standard (7), but it is not used in routine clinical practice because of the potential hazards of hypoglycemia. To evaluate adrenal responsiveness to stress, the short corticotropin test, which uses $250 \mu \mathrm{g}$ of i.v. 1-24 adrenocorticotropin (ACTH), is the one that is most commonly employed (3). The low-dose $(1 \mu \mathrm{g})$ corticotropin test has been recommended by some researchers to improve sensitivity and negative likelihood ratio in the evaluation of secondary adrenal insufficiency $(8,9,10)$, but others favor the $250 \mu \mathrm{g}$ test because of its similar performance and the availability of ready-to-use drug vials $(11,12,13$, 14). In healthy controls, both tests have been shown to elicit identical stimulated cortisol in the range observed in patients under maximum surgical stress (15).

Hypocortisolism is a potentially life-threatening condition that occurs during acute illness or in other stressful situations. When terminating glucocorticoid treatment, it is therefore common to taper the dose and instruct patients about stress prophylaxis if adrenal insufficiency is suspected or has been biochemically proven. The practice of tapering glucocorticoids is an area of uncertainty, and data from prospective studies on it are scarce. In patients that have been treated for acute exacerbations of asthma or COPD, there is no evidence of the superiority of a particular withdrawal scheme over another with respect to rebound of the disease or acute adrenal insufficiency (16). We therefore prospectively studied adrenal function and the impact of biochemically proven hypocortisolism on clinical symptoms and outcome over 6 months. We aimed to evaluate the safety of abruptly terminating systemic glucocorticoid treatment in the setting of acute exacerbations of COPD.

\section{Subjects and methods}

\section{Study design and patients}

For the present prespecified analysis, we used data from the REDUCE multicenter noninferiority trial that had previously been published $(2,17)$. In brief, patients who presented to the emergency department with acute exacerbation of COPD were included. Glucocorticoid pretreatment was defined as daily systemic therapy for 2 or more days directly preceding study inclusion (2). After 5 days of treatment with $40 \mathrm{mg}$ prednisone daily, patients were randomly allocated to continued prednisone therapy or a matching placebo for 9 days. There was no tapering after treatment was completed. The primary endpoint of the study was the time to COPD re-exacerbation during a follow-up period of 6 months. All patients were treated with a broad-spectrum antibiotic for 7 days and with the following inhaled agents from enrolment through the end of the follow-up period: Tiotropium $18 \mu \mathrm{g}$ (Spiriva $^{\mathrm{TM}}$ HandiHaler $^{\mathrm{TM}}$ ) once daily plus either Fluticasone $250 \mu \mathrm{g} /$ Salmeterol $50 \mu \mathrm{g}$ (Seretide ${ }^{\mathrm{TM}}$ spray or disc) or Budenoside $400 \mu \mathrm{g}$ plus Formoterol $12 \mu \mathrm{g}$ (Symbicort ${ }^{\mathrm{TM}}$ Turbuhaler $^{\mathrm{TM}}$ ). The trial was approved by the institutional review boards of the participating hospitals and registered with Current Controlled Trials (ISRCTN19646069). All patients provided written informed consent.

\section{Assessment of the HPA axis}

We assessed adrenal function with the low-dose $(1 \mu \mathrm{g})$ corticotropin test, which has been advocated in the evaluation of central adrenal insufficiency (9). Syringes containing $1 \mu \mathrm{g}$ of $1-24$ ACTH in $1 \mathrm{ml} 0.1$ molar acetate buffer containing albumin were prepared by the hospital pharmacy of the University Hospital Basel, Switzerland, by diluting commercially available $250 \mu \mathrm{g}$ ampoules (Synacthen $^{\mathrm{TM}}$, Novartis Pharma). The content was confirmed by liquid chromatography/mass spectrometry, 
and syringes were kept at $4{ }^{\circ} \mathrm{C}$ until use. Serum cortisol was measured in a core laboratory at the University Hospital Basel, Switzerland, by a chemoluminescence assay on a Cobas 6000 analyzer (F. Hoffmann-La Roche Ltd, Basel, Switzerland) before and $30 \mathrm{~min}$ after i.v. injection of $1 \mu \mathrm{g}$ corticotropin. Despite minimal interference with exogenous prednisone in the assay $(<1 \%$ cross-reactivity of the antibody), stimulation tests were performed $24 \mathrm{~h}$ after the last prednisone dose, just before the dose administered on the test day. Results were considered normal if at least one of the two cortisol values was $500 \mathrm{nmol} / \mathrm{l}$ or higher. Tests were performed before administering the first glucocorticoid dose in the emergency room, on day 6 before initiating blinded treatment, and on the day of discharge. The test was performed on day 30 in patients who had a biochemically suppressed HPA at discharge, and repeated tests were done on days 90 and 180 unless results became normal.

Signs and symptoms suggestive of hypocortisolism were evaluated using a modified performance score established for hypoadrenergic patients on glucocorticoid replacement therapy (18) that has been adapted to COPD patients (19). The score contained the following six items: fatigue, loss of energy, reduced strength, muscle pain, nausea, and symptoms of hypoglycemia. The item 'hyperpigmentation' was omitted from the original test version because it was not pertinent for secondary adrenal insufficiency. Each item was rated by the patient on a scale from 0 (no notice of symptom or sign) to 6 (very strong symptom or sign); the overall score thus ranged from a minimum of 0 to a maximum of 36 points.

Patients with biochemically suppressed adrenal glands at discharge were given an information sheet detailing the signs and symptoms of adrenal insufficiency, along with an emergency kit containing five $10 \mathrm{mg}$ hydrocortisone tablets. They were instructed to take $30-50 \mathrm{mg}$ of hydrocortisone and to seek professional help if symptoms suggestive of hypocortisolism occurred. Physicians involved in their care after hospital discharge were informed about the pathological results of their low-dose corticotropin tests.

\section{Statistical analysis}

Data are described using means \pm s.D., medians (quartiles), and frequencies (percentages), as appropriate. To evaluate differences between the groups, the Mann-Whitney $U$ test was used for continuous variables, and Fisher's exact test was used for categorical variables. Correlations between clinical signs of hypocortisolism and test results were measured using Spearman's rank correlation coefficient. To study associations of baseline factors with the risk for COPD re-exacerbation during follow-up, we used logistic regression models, and we report odds ratios (ORs). Logistic models were adjusted for potential confounding variables, including glucocorticoid use before hospitalization, age, GOLD COPD grade, present nicotine use, and sex. A twosided $P$ value of $<0.05$ was considered statistically significant. All calculations were performed using Stata version 12.1 (Stata Corp., College Station, TX, USA).

\section{Results}

\section{Baseline characteristics}

Of the 717 patients evaluated for eligibility, 311 had a diagnosis of acute COPD exacerbation and underwent randomization. The mean \pm s.D. age of patients was $69.8 \pm$ 10.9 years; $60.5 \%$ were male (Table 1 ). The majority of patients had severe or very severe COPD, with 32.6 and $53.8 \%$ in GOLD grades III and IV respectively. A total of $20.1 \%$ of patients had received systemic glucocorticoid pretreatment upon study entry. Among these individuals, the median daily dose at inclusion was $20 \mathrm{mg}$ prednisone equivalent.

\section{Adrenal function testing}

Upon hospital admission, corticotropin stimulation tests were performed before the first glucocorticoid dose in 236 of the 311 patients (75.9\%). It was not logistically possible to test all of the patients undergoing randomization. Basal and stimulated cortisol levels were highest on admission (496 \pm 398 and $816 \pm 413 \mathrm{nmol} / 1$ respectively) and lowest on day $6(235 \pm 174$ and $453 \pm 178 \mathrm{nmol} / \mathrm{l}$ respectively), and they showed a gradual increase thereafter (Table 2 and Fig. 1). In the complete cohort, the proportions of patients with a pathological corticotropin stimulation test at hospital discharge and on day 30 were 38 and $9 \%$ respectively, and those proportions decreased to 3 and 2\% after 90 and 180 days respectively. Logistic regression analysis showed an OR of 5.2 (95\% CI, 2.4-11.4, P<0.001) for a pathological low-dose corticotropin stimulation test at baseline (day 1) in patients who had been pretreated with glucocorticoids. The proportions of the pathological test results did not differ significantly between the treatment groups at any time point.

At hospital discharge, patients in the 5-day treatment group had significantly higher mean basal $(332 \pm 213 \mathrm{nmol} / 1$ vs $266 \pm 197 \mathrm{nmol} / 1, P=0.03)$ and stimulated $(545 \pm 209 \mathrm{nmol} / 1$ vs $475 \pm 203 \mathrm{nmol} / 1$, 
Table 1 Baseline characteristics ${ }^{a}$.

\begin{tabular}{l} 
Characteristic \\
\hline Sociodemographics \\
Age (years), mean \pm s.D. \\
Males, $n(\%)$ \\
Smoking history and lung function \\
Current smokers, $n$ (\%) \\
Pack years smoked, median (quartiles) \\
FEV $(\%$ predicted) mean \pm s.D. \\
GOLD COPD grade ${ }^{b} n(\%)$ \\
1 \\
2 \\
3 \\
4 \\
Therapy prior to presentation to the emergency room \\
Home oxygen therapy, $n$ (\%) \\
Pretreatment with systemic glucocorticoids, $n$ (\%) \\
Pretreatment daily prednisone dose (mg), median (quartiles) \\
Pretreatment with antibiotics, $n$ (\%) \\
Clinical presentation \\
Systolic blood pressure (mmHg), median (quartiles) \\
Diastolic blood pressure (mmHg), median (quartiles) \\
Heart rate (bpm), median (quartiles) \\
Oxygen saturation (\%) with nasal oxygen, median (quartiles) \\
Oxygen saturation (\%) without nasal oxygen, median (quartiles) \\
Temperature ( ${ }^{\circ} \mathrm{C}$ ), median (quartiles) \\
\hline
\end{tabular}

\begin{tabular}{|c|c|}
\hline $\begin{array}{l}\text { Overall cohort } \\
\qquad(n=311)\end{array}$ & $\begin{array}{c}\text { 14-day treatment } \\
(n=155)\end{array}$ \\
\hline $\begin{array}{l}69.8 \pm 10.9 \\
188(60.5)\end{array}$ & $\begin{array}{l}69.8 \pm 10.6 \\
83(53.5)\end{array}$ \\
\hline $\begin{array}{c}139(45.1) \\
50(30,60) \\
31.5 \pm 14.3\end{array}$ & $\begin{aligned} 62(40) \\
45(30,60) \\
31.3(13.2)\end{aligned}$ \\
\hline $\begin{array}{c}1(0.3) \\
40(13.3) \\
98(32.6) \\
162(53.8)\end{array}$ & $\begin{array}{c}0 \\
18(12.1) \\
53(35.6) \\
78(52.3)\end{array}$ \\
\hline $\begin{array}{l}40(13.0) \\
63(20.1) \\
20(5,50) \\
53(17)\end{array}$ & $\begin{array}{l}16(10.6) \\
28(18.5) \\
15(5,45) \\
21(14.0)\end{array}$ \\
\hline $\begin{array}{c}138(124,158) \\
80(70,90) \\
91(80,105) \\
95(92,97) \\
90(86,94) \\
37.3(36.8,38.1)\end{array}$ & $\begin{array}{c}138(124,158) \\
80(70,87.5) \\
90(79,105) \\
95(92,97) \\
90(85,93) \\
37.4(36.8,38.2)\end{array}$ \\
\hline
\end{tabular}

\begin{tabular}{c}
$\begin{array}{c}\text { 5-day treatment } \\
(n=156)\end{array}$ \\
\hline $69.8 \pm 11.3$ \\
$105(67.3)$ \\
$77(49.4)$ \\
$50(40,60)$ \\
$31.7(15.4)$ \\
$1(0.6)$ \\
$22(14.5)$ \\
$45(29.6)$ \\
$84(55.3)$ \\
\\
$24(15.5)$ \\
$35(22.6)$ \\
$20(10,50)$ \\
$32(21.9)$ \\
$139(124,160)$ \\
$80(71,91)$ \\
$92(80,106)$ \\
$95(92,96)$ \\
$90(86,94)$ \\
$37.3(36.7,38.0)$
\end{tabular}

$\mathrm{FEV}_{1}$, forced expiratory volume in $1 \mathrm{~s}$; GOLD, Global Initiative for Chronic Obstructive Lung Disease; bpm, beats per minute. ${ }^{a}$ Reproduced in part with permission from the Journal of the American Medical Association.

${ }^{\text {b}}$ Data on GOLD grade were available for 301 patients at baseline.

$P=0.017$ ) cortisol levels (Table 2 ). At all other time points, there were no sizeable differences in basal or stimulated cortisol levels between the two steroid exposure groups.

\section{Association of cortisol levels with clinical symptoms}

To evaluate the associations of cortisol levels with clinical symptoms, we correlated basal and stimulated values at various time points with symptoms of adrenal insufficiency using a modified score derived from a standardized questionnaire $(18,19)$. A higher score indicated more symptoms of hypocortisolism. In general, cortisol levels correlated weakly or not at all with any individual clinical item at the different time points (Table 3). Basal and stimulated cortisol values did not correlate consistently with any single item or the total points in the adrenal insufficiency score across the various time points. Moreover, correction for multiple testing resulted in statistical significance at a $P$ value of $<0.0006$. At this level, none of the correlations was statistically significant.

We also compared clinical symptoms of hypocortisolism in patients with normal and pathological low-dose corticotropin test at each time point, considering the complete cohort and patients with 5-day and 14-day glucocorticoid therapy separately. No statistically significant difference could be detected, regardless of the treatment duration (Fig. 2A, B, and C). Thus, biochemical suppression of the HPA axis did not translate into clinically detectable adrenal insufficiency.

\section{Associations of adrenal function with risk for adrenal crisis, COPD re-exacerbation, and glucocorticoid exposure after hospital discharge}

During the follow-up of 180 days, no patients needed treatment for adrenal crisis, despite the high proportion of patients with impaired adrenal function, in particular at hospital discharge.

We investigated the association between adrenal function, as assessed biochemically at various time points, and the later risk for COPD re-exacerbation using a logistic regression model adjusted for glucocorticoid use before hospitalization, age, COPD severity as reflected by GOLD grade, active nicotine consumption, and sex (Table 4). Overall, basal and stimulated cortisol levels were inversely associated with re-exacerbation risk, whereas a 
pathological low-dose corticotropin test was associated with higher odds for re-exacerbation. The numbers reached statistical significance on day 6 , when an insufficient adrenal response to corticotropin was associated with an OR of $2.21(P=0.006)$, and higher basal and stimulated cortisol levels were associated with a significantly lower risk for re-exacerbation (OR: 0.27 and 0.09, $P=0.001$ and 0.001 respectively). We also conducted a survival analysis for time to re-exacerbation using a proportional hazard model that included the same covariates as our primary logistic regression model but considered stimulated cortisol levels assessed on day 6, at discharge, and on days 30 and 90 as time-dependent covariate. Consistent with the logistic regression, we found a significant negative association between stimulated cortisol and risk of re-exacerbation; a doubling of the stimulated cortisol level was associated with a hazard ratio of 0.65 (95\% CI, 0.47-0.90, $P=0.01$ ). Patients treated with glucocorticoid before admission to the emergency room were prescribed significantly higher mean cumulative steroid doses after hospital discharge until the end of follow-up (433 \pm 73 vs $150 \pm 26 \mathrm{mg}$ prednisone, mean difference $284 \mathrm{mg}$ (95\% CI, 408-159), $P<0.0001)$. In patients with a pathological corticotropin test at hospital discharge, there was a trend toward a higher glucocorticoid exposure during follow-up than that seen in patients with a normal test (305 $\mathrm{mg}$ prednisone equivalent vs $196 \mathrm{mg}$ respectively; mean absolute difference: $109 \mathrm{mg}$ (95\% CI, -41 to 259 ), $P=0.15$ ).

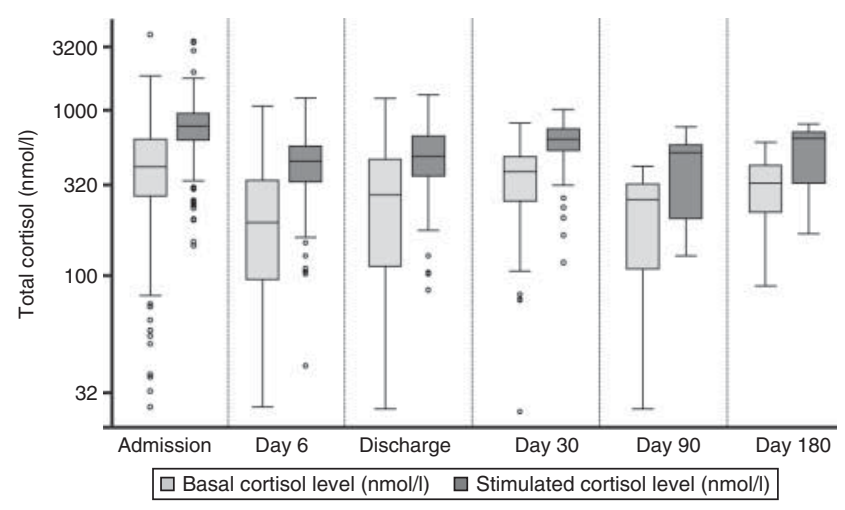

Figure 1

Cortisol levels at different time points. Values are shown for the whole cohort. Boundaries of the boxes show the interquartile range (IQR); the line in the box shows the median. The length of the whiskers denotes $1.5 \times$ the IQR. Circles show outlier values. 


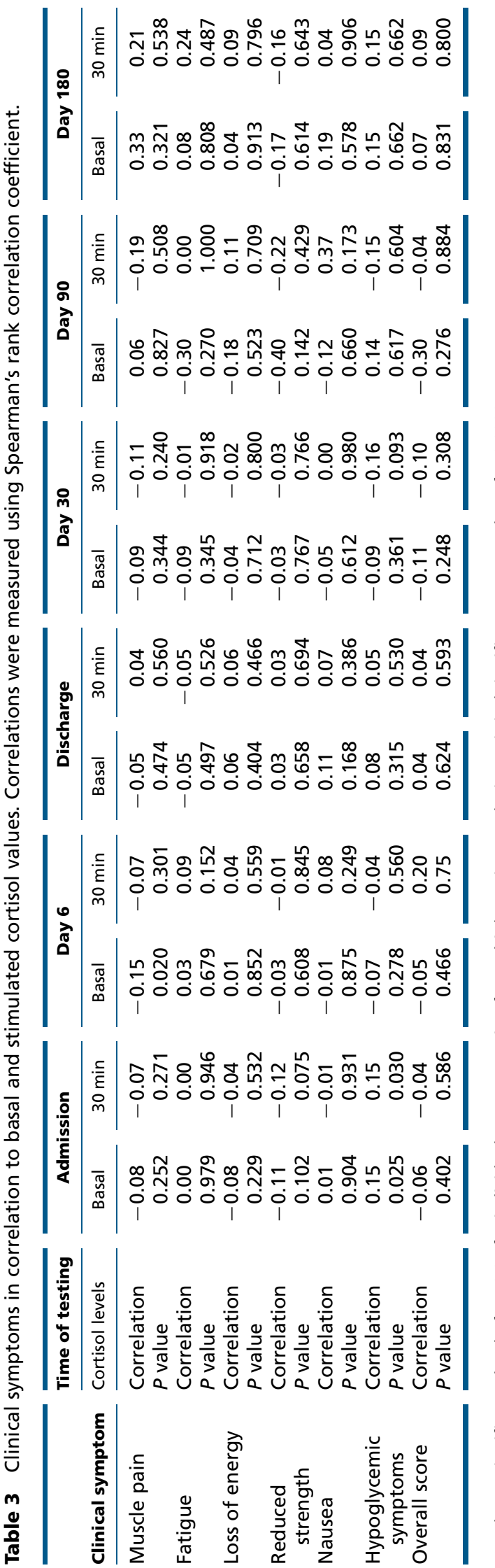

\section{Discussion}

The REDUCE randomized controlled trial (2) longitudinally assessed adrenal function in patients with acute COPD exacerbation allocated to conventional (14-day) or short (5-day) treatment with $40 \mathrm{mg}$ prednisone daily. At study entry, $15 \%$ of patients presented with adrenal insufficiency, as judged by the low-dose $(1 \mu \mathrm{g})$ corticotropin test; of these, $45 \%$ had been pretreated with systemic glucocorticoids (compared to only $14 \%$ in patients with a normal low-dose corticotropin test) (2). After 5 days of systemic glucocorticoid treatment, $63 \%$ of patients showed a biochemically suppressed adrenal gland. This finding is in accordance with earlier data, which showed that short-term (5-20 days of) therapy with $\geq 25 \mathrm{mg}$ prednisone equivalent dailyled to biochemical adrenal suppression in $\sim 45-90 \%$ of subjects $(6,19)$. In the present study, 38\% of our patients were discharged from the hospital with a pathological low-dose corticotropin test. This proportion was the same in the short-term and conventional therapy groups, despite differing treatment durations and differences in median length of hospital stay (8 days (interquartile range (IQR): $5-11$ ) in the shortterm and 9 days (IQR: 6-14) in the conventional group respectively (2)). At no time point did we find significant differences in the percentage of pathological stimulation test results between the groups, although the cumulative prednisone doses in the short-term and conventional treatment groups after 6 months differed significantly (mean: 379 vs $793 \mathrm{mg}$ at the end of follow-up respectively; difference in means: $-414 \mathrm{mg}$ (95\% CI, -521 to -307 , $P \leq 0.001)$ ) (2). Our findings support the notion that the cumulative glucocorticoid dose does not predict adrenal function or the pace of recovery following suppression by exogenous glucocorticoids $(5,6)$.

Interestingly, we found a significant inverse correlation between cortisol levels, measured on day 6 and after, and the risk of COPD re-exacerbation during the 6-month follow-up. This is, to our knowledge, a novel finding. It is conceivable that lower endogenous cortisol levels (i.e., persistent suppression of the circadian rhythm of the HPA axis) could mirror weaker antiinflammatory activity and thus could have led to an increased risk of re-exacerbation. Alternatively, more frequent re-exacerbations could have led to increased glucocorticoid use and thus suppression of the HPA axis. Our data do not permit us to define the causality of our observation, but they do underscore the notion that glucocorticoid exposure should be minimized in patients with COPD. When comparing symptoms of 

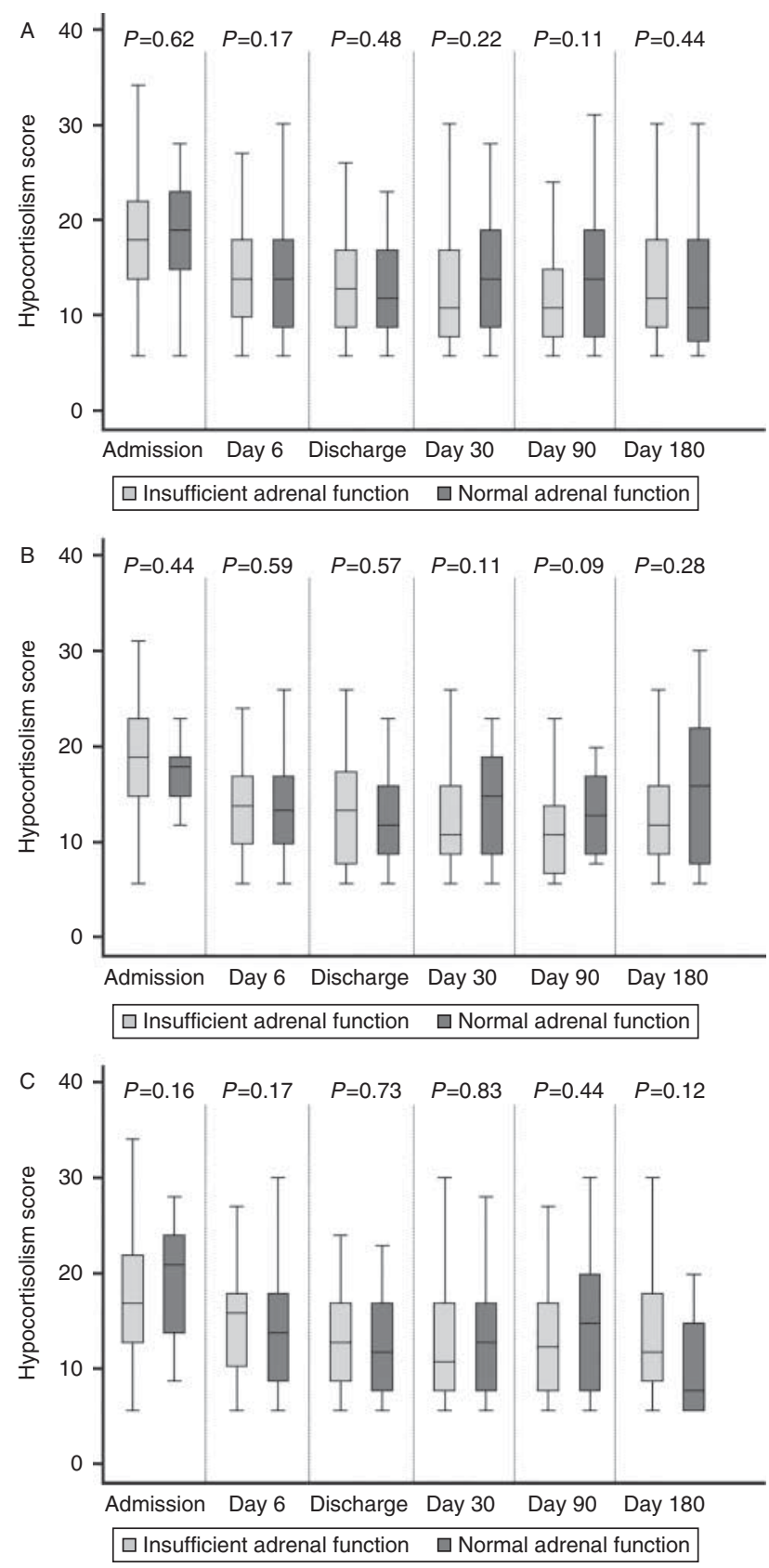

\section{Figure 2}

Clinical hypocortisolism score (range: $0-36$ points) in patients with normal and pathological low-dose corticotropin stimulation tests at different time points. (A) Complete cohort.

(B) Patients with 14-day glucocorticoid treatment.

(C) Patients with 5-day glucocorticoid treatment.

hypocortisolism in patients with biochemically normal and pathological adrenal function across all time points, we found no differences. This result confirms earlier findings from a smaller study (19) and suggests that the

low-dose corticotropin test for clinically relevant cortisol deficiency may yield false pathologic results in the setting of acute COPD exacerbation. Thus, our data do not support cortisol measurements before and after the treatment of exacerbated COPD outside the context of clinical trials.

When systemic glucocorticoid therapy is rapidly discontinued, a rebound of the treated inflammatory disease, specific signs and symptoms of hypocortisolism, or an unspecific withdrawal syndrome may theoretically occur alone or in combination (20). In clinical practice, physicians use corticotropin stimulation tests to evaluate the indication of replacement therapy or to guide tapering after glucocorticoid treatment (21). Whether this approach is justified, particularly in the absence of clinical symptoms of hypocortisolism, is unclear, seeing as in acute illness, mechanisms other than ACTH stimulation have been shown to be involved in providing adequate cortisol levels (22). Multiple glucocorticoid tapering schemes are in use, but randomized controlled trials are rare. A small study in patients discharged from the hospital after glucocorticoid treatment for acute asthma attacks found no differences in lung function between patients randomized to either abrupt stopping or tapering prednisolone over 2 weeks (23). An analysis that included nine randomized controlled trials in which patients were withdrawn from systemic glucocorticoid treatment for acute exacerbations of chronic disorders found no difference in adverse effects between withdrawal regimens (16). We stopped prednisone treatment without tapering irrespective of glucocorticoid therapy before study entry and irrespective of adrenal function at any time point throughout the trial, but we provided patients with pathological corticotropin tests at hospital discharge and thereafter with instructions on emergency replacement therapy. Despite the abrupt discontinuation of prednisone even in patients with a biochemically suppressed HPA axis, none of the patients experienced a hypoadrenergic crisis. This suggests that in the setting of acute exacerbation of COPD, tapering is not needed even after 14 days of treatment with prednisone. Nevertheless, our findings should not mislead clinicians to underestimate the dangers of adrenal crisis in situations of greater stress than those that occurred in the present study.

The present study has several limitations. The protocol did not plan to detect specific indications for glucocorticoid medication during the follow-up period apart from COPD re-exacerbation. We therefore cannot specify which patients were given glucocorticoids for stress prophylaxis or whether cumulative glucocorticoid 
Table 4 Association of the low-dose corticotropin test and adjusted risk for COPD re-exacerbation. ORs refer to the change in risk associated with the respective risk factor and were estimated using logistic regression models with adjustment for glucocorticoid use before hospitalization, age, COPD severity (as assessed by GOLD), active nicotine consumption and sex. Cortisol levels (nmol/l) were log-transformed. ORs associated with cortisol levels correspond to a tenfold increase in cortisol concentration and refer to logistic regression models adjusted for glucocorticoid use prior to hospitalization, age, COPD severity (according to GOLD grade), active nicotine consumption and sex.

\begin{tabular}{l}
\hline \\
\hline Day 6 \\
Pathological low-dose stimulation test \\
Baseline cortisol level \\
Stimulated cortisol level \\
Hospital discharge \\
Pathological low-dose stimulation test \\
Baseline cortisol level \\
Stimulated cortisol level \\
Day 30 \\
Pathological low-dose stimulation test \\
Baseline cortisol level \\
Stimulated cortisol level \\
Day 90 \\
Pathological low-dose stimulation test \\
Baseline cortisol level \\
Stimulated cortisol level \\
\hline
\end{tabular}

\begin{tabular}{c}
\hline OR $(95 \% \mathrm{Cl})$ \\
\hline \\
$2.21(1.26,3.86)$ \\
$0.27(0.13,0.58)$ \\
$0.09(0.02,0.4)$ \\
\\
$1.19(0.71,1.99)$ \\
$0.54(0.27,1.1)$ \\
$0.49(0.12,1.89)$ \\
\\
$1.52(0.74,3.11)$ \\
$0.46(0.15,1.38)$ \\
$0.36(0.05,2.77)$ \\
\\
$1.07(0.39,2.91)$ \\
$0.55(0.14,2.19)$ \\
$0.56(0.04,7.03)$
\end{tabular}

\begin{tabular}{c}
\hline P value \\
\hline \\
0.006 \\
0.001 \\
0.001 \\
\\
0.503 \\
0.091 \\
0.298 \\
\\
0.254 \\
0.166 \\
0.328 \\
\\
0.896 \\
0.399 \\
0.653
\end{tabular}

$\mathrm{OR}$, odds ratio. $\mathrm{Cl}$, confidence interval.

stress doses were associated with performance in the corticotropin stimulation test. We did observe a trend toward higher total glucocorticoid exposure after hospital discharge until the end of follow-up in patients with a pathological test at discharge. However, we cannot formally prove a causal relation of this finding to the higher risk of COPD re-exacerbation in patients with lower cortisol values. Also, our protocol was not designed to assess which drugs patients were taking across different time points (apart from those related to COPD management). To assess the signs and symptoms of cortisol deficiency, we used a modified clinical score originally developed for patients with primary and secondary adrenal insufficiency on cortisone replacement therapy (18). Although this score has been used in patients with COPD exacerbations (19), it was not designed and has not been formally validated for this clinical setting. Also, it does not specifically detect mental symptoms of glucocorticoid deficiency, although we did not observe any patients with a clinically relevant steroid withdrawal syndrome. Our findings suggest that the signs and symptoms of the score are not specific enough to clinically detect biochemically proven hypocortisolism.

In conclusion, early biochemical suppression of the adrenal glands occurred in about two-thirds of patients treated for acute COPD exacerbation. A pathological low-dose $(1 \mu \mathrm{g})$ corticotropin test did not translate into clinical hypocortisolism, but it did reflect a higher risk for COPD re-exacerbation. In patients treated for exacerbated COPD, abruptly stopping glucocorticoids while also providing emergency hydrocortisone tablets and adequate instructions for their use was safe even in patients with biochemically suppressed adrenal glands.

\section{Declaration of interest}

J D Leuppi received grants from Astra Zeneca and Boehringer Ingelheim. He also serves as a board member for Novartis, consulted for Merck, Sharp \& Dohme Chibret, received payment from Boehringer Ingelheim for lectures, and received payment from Astra Zeneca for developing educational presentations. P Schuetz received consulting and lecture fees from Thermo Fisher Scientific and bioMerieux. $R$ Bingisser serves as a board member of Daiichi Sankyo, Eli Lilly, Merck, Sharp \& Dohme Chibret, GE, Phillipps, and Sanofi-Aventis. He also received grants from Bayer, Pfizer, and BRAHMS, and he received payment from Astra Zeneca for lectures. $C$ von Garnier serves as an advisory board member for Takeda Pharma and Boehringer Ingelheim (Switzerland). J Rutishauser received grants from Astra Zeneca and Viollier Laboratories.

\section{Funding}

The REDUCE trial was an investigator-initiated study supported by: the Departments of Medicine, University Hospital Basel and Hospital Centre, Biel/Bienne; Freiwillige Akademische Gesellschaft, Basel; Fonds für Lehre und Forschung, Basel; Astra Zeneca Switzerland, Inc., Zug; Viollier Laboratories, Allschwil; and Gottfried und Julia Bangerter-Rhyner, Stiftung für Medizinische Forschung, Bern (all Switzerland). None of the sponsors 
had any role in the design or conduct of the study, the collection, management, analysis, or interpretation of the data, or the preparation, review, or approval of the manuscript.

\section{Author contribution statement}

Study design: P Schuetz, J D Leuppi, R Bingisser, M Briel, B Mueller, C Schindler, M Tamm, J Rutishauser; analysis and interpretation of data: P Schuetz, C Schindler, J Rutishauser; drafting of the manuscript: P Schuetz, J Rutishauser; revision of the manuscript: $P$ Schuetz, J D Leuppi, R Bingisser, M Bodmer, M Briel, T Drescher, C Henzen, D Miedinger, B Mueller, A Scherr, C Schindler, R Stoeckli, S Viatte, C von Garnier, M Tamm, J Rutishauser, U Duerring, Y Leibbrandt, S Maier; statistical analysis: P Schuetz, C Schindler; study nurses: U Duerring, Y Leibbrandt, S Maier; principal investigator: J Rutishauser.

\section{Acknowledgements}

We thank Dr Bruno Allolio for his helpful comments on the manuscript draft.

\section{References}

1 Global strategy for the diagnosis, management and prevention of COPD. Global Initiative for Chronic Obstructive Lung Disease (GOLD), updated January 2015. www.goldcopd.org.

2 Leuppi JD, Schuetz P, Bingisser R, Bodmer M, Briel M, Drescher T, Duerring U, Henzen C, Leibbrandt Y, Maier S et al. Short-term vs conventional glucocorticoid therapy in acute exacerbations of chronic obstructive pulmonary disease: the REDUCE randomized clinical trial. Journal of the American Medical Association 2013309 2223-2231. (doi:10.1001/jama.2013.5023)

3 Arlt W \& Allolio B. Adrenal insufficiency. Lancet 2003361 1881-1893. (doi:10.1016/S0140-6736(03)13492-7)

4 Sacre K, Dehoux M, Chauveheid MP, Chauchard M, Lidove O, Roussel R \& Papo T. Pituitary-adrenal function after prolonged glucocorticoid therapy for systemic inflammatory disorders: an observational study. Journal of Clinical Endocrinology and Metabolism 201398 3199-3205. (doi:10.1210/jc.2013-1394)

5 Schlaghecke R, Kornely E, Santen RT \& Ridderskamp P. The effect of long-term glucocorticoid therapy on pituitary-adrenal responses to exogenous corticotropin-releasing hormone. New England Journal of Medicine 1992326 226-230. (doi:10.1056/NEJM199201233260403)

6 Henzen C, Suter A, Lerch E, Urbinelli R, Schorno XH \& Briner VA. Suppression and recovery of adrenal response after short-term, highdose glucocorticoid treatment. Lancet 2000355 542-545. (doi:10.1016/ S0140-6736(99)06290-X)

7 Jones SL, Trainer PJ, Perry L, Wass JA, Bessser GM \& Grossman A. An audit of the insulin tolerance test in adult subjects in an acute investigation unit over one year. Clinical Endocrinology $1994 \mathbf{4 1}$ 123-128. (doi:10.1111/j.1365-2265.1994.tb03793.x)

8 Dickstein G, Shechner C, Nicholson WE, Rosner I, Shen-Orr Z, Adawi F $\&$ Lahav M. Adrenocorticotropin stimulation test: effects of basal cortisol level, time of day, and suggested new sensitive low dose test. Journal of Clinical Endocrinology and Metabolism 199172 773-778. (doi:10.1210/jcem-72-4-773)
9 Thaler LM \& Blevins LS Jr. The low dose (1-microg) adrenocorticotropin stimulation test in the evaluation of patients with suspected central adrenal insufficiency. Journal of Clinical Endocrinology and Metabolism 199883 2726-2729.

10 Kazlauskaite R, Evans AT, Villabona CV, Abdu TA, Ambrosi B, Atkinson $\mathrm{AB}$, Choi $\mathrm{CH}$, Clayton RN, Courtney CH, Gonc EN et al. Corticotropin tests for hypothalamic-pituitary-adrenal insufficiency: a metaanalysis. Journal of Clinical Endocrinology and Metabolism 200893 4245-4253. (doi:10.1210/jc.2008-0710)

11 Mayenknecht J, Diederich S, Bahr V, Plockinger U \& Oelkers W. Comparison of low and high dose corticotropin stimulation tests in patients with pituitary disease. Journal of Clinical Endocrinology and Metabolism 199883 1558-1562. (doi:10.1210/jcem.83.5.4831)

12 Dorin RI, Qualls CR \& Crapo LM. Diagnosis of adrenal insufficiency. Annals of Internal Medicine 2003139 194-204. (doi:10.7326/0003-4819139-3-200308050-00017)

13 Stewart PM \& Clark PM. The low-dose corticotropin-stimulation test revisited: the less, the better? Nature Clinical Practice. Endocrinology \& Metabolism 20095 68-69. (doi:10.1038/ncpendmet1038)

14 Oelkers W. M. Pura et al. (Lubochna and Prague) with the title: "The low dose (1 microg) cosyntropin test (LDT) for primary adrenocortical insufficiency: defining the normal cortisol response and report on first patients with Addison disease confirmed with LDT" (ECED ; 118: 151-157). Experimental and Clinical Endocrinology \& Diabetes 2010118 339-340. (doi:10.1055/s-0030-1253438)

15 Widmer IE, Puder JJ, König C, Pargger H, Zerkowski HR, Girard J \& Müller B. Cortisol response in relation to the severity of stress and illness. Journal of Clinical Endocrinology and Metabolism 200590 4579-4586. (doi:10.1210/jc.2005-0354)

16 Richter B, Neises G \& Clar C. Glucocorticoid withdrawal schemes in chronic medical disorders. A systematic review. Endocrinology and Metabolism Clinics of North America 200231 751-778. (doi:10.1016/ S0889-8529(02)00008-7)

17 Schuetz P, Leuppi JD, Tamm M, Briel M, Bingisser R, Dürring U, Müller B, Schindler C, Viatte S \& Rutishauser J. Short versus conventional term glucocorticoid therapy in acute exacerbation of chronic obstructive pulmonary disease - the "REDUCE" trial. Swiss Medical Weekly 2010140 w13109.

18 Arlt W, Rosenthal C, Hahner S \& Allolio B. Quality of glucocorticoid replacement in adrenal insufficiency: clinical assessment vs timed serum cortisol measurements. Clinical Endocrinology 200664 384-389.

19 Schuetz P, Christ-Crain M, Schild U, Suess E, Facompre M, Baty F, Nusbaumer C, Brutsche M \& Muller B. Effect of a 14-day course of systemic corticosteroids on the hypothalamic-pituitary-adrenal-axis in patients with acute exacerbation of chronic obstructive pulmonary disease. BMC Pulmonary Medicine 20088 1. (doi:10.1186/1471-2466-8-1)

20 Hochberg Z, Pacak K \& Chrousos GP. Endocrine withdrawal syndromes. Endocrine Reviews 200324 523-538. (doi:10.1210/er.2001-0014)

21 Kountz DS \& Clark CL. Safely withdrawing patients from chronic glucocorticoid therapy. American Family Physician 199755 521-525, 529-530.

22 Boonen E, Vervenne H, Meersseman P, Andrew R, Mortier L, Declercq PE, Vanwijngaerden Y-M, Spriet I, Wouters PJ, Vander Perre S et al. Reduced cortisol metabolism during critical illness. New England Journal of Medicine 2013368 1477-1488. (doi:10.1056/ NEJMoa1214969)

23 Hatton MQ, Vathenen AS, Allen MJ, Davies S \& Cooke NJ. A comparison of 'abruptly stopping' with 'tailing off' oral corticosteroids in acute asthma. Respiratory Medicine 199589 101-104. (doi:10.1016/ 0954-6111(95)90191-4)

Received 16 February 2015

Revised version received 31 March 2015

Accepted 8 April 2015 